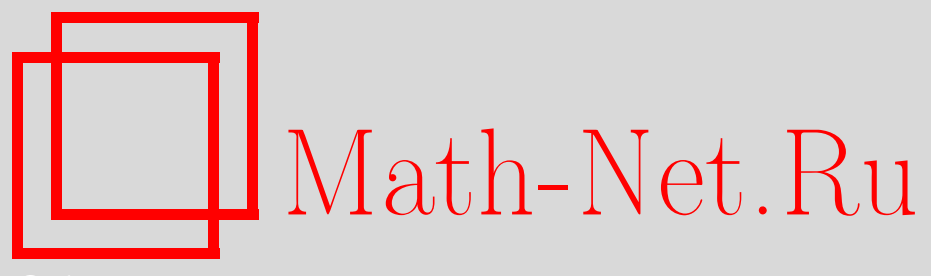

А. А. Арсеньев, О представлении функции Грина уравнения Шрёдингера с почти периодическим потенциалом континуальным интегралом по когерентным состояниям, Матем. сб., 2006, том 197, номер 11, 3-12

DOI: https://doi.org/10.4213/sm1486

Использование Общероссийского математического портала Math-Net.Ru подразумевает, что вы прочитали и согласны с пользовательским соглашением http://www . mathnet.ru/rus/agreement

Параметры загрузки:

IP : 3.85 .183 .62

26 апреля 2023 г., 15:45:50

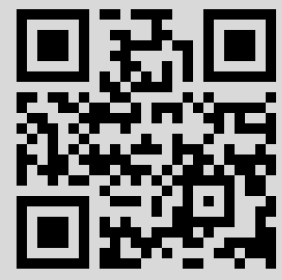




\section{А. А. Арсеньев \\ О представлении функции Грина уравнения Шрёдингера с почти периодическим потенциалом континуальным интегралом по когерентным состояниям}

В статье рассматривается задача Коши для многомерного уравнения Шрёдингера, описывающего частицу в постоянном электрическом и магнитном полях и поле потенциала, который есть сумма убывающей и почти периодической функций. Предложена аппроксимация функции Грина задачи Коши для такого уравнения континуальным интегралом по гауссовым волновым пакетам.

Библиография: 21 название.

\section{§1. Постановка задачи}

Векторы ориентированного трехмерного евклидова пространства $\mathbb{R}^{3}$ будем обозначать символами $x, y \ldots$. Скалярное произведение в $\mathbb{R}^{3}$ обозначим символом $(\cdot, \cdot)$, векторное произведение векторов $h$ и $x-$ символом $h * x$. Символ $f(x)$ в зависимости от контекста означает имя функции, значение функции в точке или оператор умножения на функцию. Для матрицы $C$ с комплексными элементами мы по определению положим

$$
(x, C y)=(x,(\operatorname{Re} C) y)+i(x,(\operatorname{Im} C) y) .
$$

На функциях из $C_{0}^{\infty}\left(\mathbb{R}^{3}\right)$ определим оператор $H$ :

$$
H \psi(x)=\frac{1}{2}\left(-i \hbar \partial_{x}-\mathscr{A}(x)\right)^{2} \psi(x)+((l, x)+v(x)) \psi(x),
$$

где

$$
\mathscr{A}(x)=\frac{1}{2} h * x, \quad \hbar>0, \quad h, l \in \mathbb{R}^{3}
$$

и $v(x)$ - действительная функция (потенциал). Мы будем предполагать, что потенциал $v(x)$ представим в виде суммы двух действительных функций:

$$
v(x)=v_{\text {ext }}(x)+v_{\text {bulk }}(x),
$$

где

$$
\begin{aligned}
v_{\text {ext }}(x) & =\int \exp (i(x, k)) \widehat{v(k)} d k, \\
v_{\text {bulk }}(x) & =\sum_{j} a(j) \exp (i(b(j), x)), \quad j \in \mathbb{Z}, \quad b(j) \in \mathbb{R}^{3},
\end{aligned}
$$


причем

$$
\operatorname{norm}(v) \stackrel{\text { def }}{=} \int(1+|k|)^{3}|\widehat{v(k)}| d k+\sum_{j}(1+|b(j)|)^{3}|a(j)|<\infty .
$$

Если векторы $b(j)$ в $(3 \mathrm{~b})$ пробегают периодическую решетку в $\mathbb{R}^{3}$, то потенциал $v_{\text {bulk }}(x)$ будет периодичен.

Далее тем же символом $H$ мы будем обозначать самосопряженное в $L^{2}\left(\mathbb{R}^{3}\right)$ расширение оператора (1).

Рассмотрим задачу Коши

$$
i \hbar \partial_{t} \psi(x, t)=H \psi(x, t), \quad \psi(x,+0)=\psi_{0}(x) .
$$

Задачу (5) мы будем рассматривать как абстрактную задачу Коши в $L^{2}\left(\mathbb{R}^{3}\right)$ и будем рассматривать ее на произвольном, но фиксированном интервале времени $T$. В квантовой механике задача (5) описывает эволюцию волновой функции частицы, которая движется в магнитном поле $h$, электрическом поле $l$ и поле с потенциалом $v(x)$. В математических задачах физики твердого тела потенциал $v_{\text {bulk }}(x)$ обычно описывает взаимодействие частицы с атомами образца, а потенциал $v_{\text {ext }}(x)$ описывает внешнее взаимодействие.

Наша цель состоит в том, чтобы дать представление функции Грина задачи (5) в виде континуального интеграла.

В физической литературе под термином "континуальный интеграл" понимают предел $n$-кратных интегралов при $n \rightarrow \infty$. Обычно в физике континуальные интегралы используются как вспомогательное средство при построении формальных рядов теории возмущений, однако есть примеры эффективного использования континуальных интегралов при численных расчетах. Техника континуальных интегралов стала популярной после работ физика Р. Фейнмана, поэтому континуальные интегралы иногда называют фейнмановскими интегралами или интегралами по путям. Для построения функции Грина задачи Коши континуальные интегралы использовались в работах [1], [2]. В своих построениях мы используем когерентные состояния (которые в рассматриваемом нами случае есть гауссовы волновые пакеты) и преобразование Фурье-Гаусса (FBI-преобразование в терминологии [3]). Теория континуальных интегралов по когерентным состояниям обсуждается в [4], [5]. Теория гауссовых волновых пакетов рассматривается в работах [4], [6]-[14]. Ссылки на работы по применению FBI-преобразования к исследованию уравнения Шрёдингера можно почерпнуть в [15], [16].

Основной результат нашей работы сформулирован в теореме 1. Обратим внимание на следующие особенности оценки (40):

1) оценка (40) равномерна по квазиклассическому параметру $\hbar$, и остаточный член в этой оценке стремится к нулю при $\hbar \rightarrow 0$;

2) при фиксированном значении параметра $\hbar$ остаточный член в оценке (40) стремится к нулю при $n \rightarrow \infty$ быстрее, чем можно было бы ожидать на основе формулы Троттера; в связи с этим интересно сравнить оценку (40) с оценками в работе [17];

3) для получения оценки (40) мы не требуем бесконечной гладкости потенциала, что обычно используется при традиционной технике ПДО. 
Используемая нами схема вычислений имеет много общего с техникой, традиционно применяемой для вычисления квазиклассической асимптотики. По применяемой в теории квазиклассики терминологии нашу основную теорему 1 можно назвать теоремой типа Егорова, поскольку она описывает эволюцию квантово-механической системы в терминах классического гамильтонова потока. Однако по следующим причинам оценка (40) не является в полном смысле квазиклассической:

1) за счет выбора параметра $n$ остаточный член в оценке (40) может быть сделан малым и при $\hbar \approx 1$, и в этом смысле оценка (40) имеет более общую область применения, чем квазиклассика;

2) мы не вычисляем асимптотику по параметру $\hbar$ входящего в оценку (40) $n$-кратного интеграла, а эта задача может быть сложной.

Обычно при построении функции Грина или параметрикса для уравнения Шрёдингера также используют приближения $n$-кратными интегралами при $n \rightarrow \infty$, а метод континуальных интегралов отличается тем, что он основан либо на идеях, связанных с формулой Троттера, либо (как в нашем случае) на идеях, связанных с обобщением метода Эйлера.

\section{§ 2. Вспомогательные построения}

Положим

$$
V(Q)=\frac{1}{2} \mathscr{A}(Q)^{2}+(l, Q)+v(Q)
$$

Пусть

$$
Q \mapsto D V(Q), \quad Q \mapsto D^{2} V(Q)
$$

векторное поле и поле операторов, которые соответствуют первому и второму дифференциалу функции (6). Квантовомеханическому гамильтониану (1) соответствует классический гамильтониан

$$
\mathscr{H}(P, Q)=\frac{1}{2}(P-\mathscr{A}(Q))^{2}+(l, Q)+v(Q), \quad P, Q \in \mathbb{R}^{3} .
$$

Уравнения движения классической динамической системы с гамильтонианом (7) суть:

$$
\begin{array}{llrl}
\partial_{t} Q & =P-\mathscr{A}(Q), & & Q\left(0, q_{0}, p_{0}\right)=q_{0}, \\
\partial_{t} P=-\mathscr{A}(P)-D V(Q), & & P\left(0, q_{0}, p_{0}\right)=p_{0} .
\end{array}
$$

В дальнейшем символами $P, Q$ мы будем обозначать решения системы (8), рассматриваемые как функции начальных данных $q_{0}, p_{0}$.

Положим

$$
V_{2}(Q, x)=V(Q)+((x-Q), D V(Q))+\frac{1}{2}\left((x-Q), D^{2} V(Q)(x-Q)\right) .
$$

Рассмотрим задачу Коши

$$
\begin{aligned}
i \hbar \partial_{t} W\left(x, t, q_{0}, p_{0}\right) & =\left(-\frac{1}{2} \hbar^{2} \Delta_{x}+i \hbar \mathscr{A}(x) \cdot \partial_{x}+V_{2}(Q, x)\right) W\left(x, t, q_{0}, p_{0}\right), \\
W\left(x,+0, q_{0}, p_{0}\right) & =(2 \pi \hbar)^{-3}(\pi \hbar \sigma)^{-3 / 2} \exp \left(-\frac{\left(x-q_{0}\right)^{2}}{2 \hbar \sigma}+i \frac{\left(p_{0},\left(x-q_{0}\right)\right)}{\hbar}\right) .
\end{aligned}
$$


Лемма 1. Решение задачи (9) дается формулой

$$
\begin{aligned}
W\left(x, t, q_{0},\right. & \left.p_{0}\right)=(2 \pi \hbar)^{-3}(\pi \hbar)^{-3 / 2}(\operatorname{det}(A))^{-1 / 2} \\
& \times \exp \left(-\frac{((x-Q), C(t)(x-Q)) / 2-i(P,(x-Q))-i S(t)}{\hbar}\right),
\end{aligned}
$$

где $P, Q$ - решение системы (8), $C(t)$ вычисляется по формулам (14) и (17), матрицы $A(t), B(t)$ вычисляются как решения системы (18), функция $S(t)$ вычисляется по формуле (21).

ДоказАТЕльство. Решение задачи (9) можно вычислить по приведенным в [9], [13] формулам, однако преобразование этих формул к удобному для нашего анализа виду сложно, поэтому мы поступим так: будем искать решение уравнения (9а) в виде

$$
\psi(x, t)=\exp \left(-\frac{((x-Q), C(t)(x-Q)) / 2-i(P,(x-Q))-i \gamma(t)}{\hbar}\right),
$$

где $C(t)$ - неизвестная матрица, а $\gamma(t)$ - неизвестная функция. Подставляя (11) в (9a), мы получаем уравнения

$$
\begin{gathered}
\partial_{t} C(t)+\frac{1}{2}(h * C(t)-C(t) h *)+i C(t)^{2}-i D^{2} V(Q)=0, \\
\partial_{t} \gamma(t)-\frac{1}{2} P^{2}+V(Q)+\frac{1}{2} \hbar \cdot \operatorname{trace}(C(t))=0,
\end{gathered}
$$

где через $h *$ мы обозначили оператор в $\mathbb{R}^{3}$ :

$$
h *: x \mapsto h * x .
$$

В уравнении (12) сделаем замену

$$
C(t)=\exp \left(-\frac{1}{2} t h *\right) Z(t) \exp \left(\frac{1}{2} t h *\right) .
$$

Для матрицы $Z(t)$ получим уравнение Риккати

$$
\begin{aligned}
& \partial_{t} Z(t)+i Z^{2}(t)=M(t), \quad Z(0)=\frac{1}{\sigma} E \\
& M(t)=\exp \left(\frac{1}{2} t h *\right) D^{2} V(Q) \exp \left(-\frac{1}{2} t h *\right) .
\end{aligned}
$$

Уравнение (15) линеаризуем подстановкой

$$
Z(t)=B(t) A^{-1}(t) .
$$

Для матриц $A(t)$ и $B(t)$ получаем уравнения

$$
\begin{array}{ll}
\partial_{t} A(t)=i B(t), & A(0)=\sigma E, \\
\partial_{t} B(t)=i M(t) A(t), & B(0)=E,
\end{array}
$$


где функция $M(t)$ определена в (16). Из теоремы Лиувилля и (18) следует, что

$$
\operatorname{trace}(C(t))=\operatorname{trace}\left(B(t) A^{-1}(t)\right)=-i \partial_{t} \ln \operatorname{det}(A(t)),
$$

поэтому с учетом начальных условий $(9 \mathrm{~b})$

$$
\gamma(t)=S(t)+i \frac{1}{2} \hbar \ln \operatorname{det}(A(t))
$$

где

$$
S(t)=\int_{0}^{t}\left(\frac{1}{2} P^{2}(\tau)-V(Q(\tau))\right) d \tau
$$

Лемма доказана.

В дальнейшем символом $а$ будем обозначать положительную константу, значение которой произвольно, но фиксировано на протяжении всей работы, символами const, $\delta$ мы обозначим положительные константы, значения которых может меняться при каждом вхождении в формулу.

ЛЕмма 2. На интервале $0 \leqslant t \leqslant a \sigma$ в евклидовой норме справедливы оценки

$$
\begin{gathered}
A(t)=(\sigma+i t) E+O\left(\sigma^{3}\right), \quad B(t)=E+O\left(\sigma^{2}\right), \quad C(t)=(\sigma+i t)^{-1}\left(E+O\left(\sigma^{2}\right)\right), \\
(E+\sigma C(t))^{-1}=\left(\frac{2 \sigma^{2}+t^{2}+i \sigma t}{4 \sigma^{2}+t^{2}}\right)\left(E+O\left(\sigma^{2}\right)\right) .
\end{gathered}
$$

ДокАзАтЕльство. Из (18) следует, что матрица $A(t)$ удовлетворяет интегральному уравнению

$$
A(t)=(\sigma+i t) E-\int_{0}^{t}(t-\tau) M(\tau) A(\tau) d \tau
$$

Из этого интегрального уравнения и неравенства Гронуолла следует оценка

$$
\|A(t)\| \leqslant \text { const } \cdot \sigma .
$$

Подставляя эту оценку в интегральное уравнение, получаем оценку матрицы $A(t)$. Подставляя оценку матрицы $A(t)$ в уравнение $(18 \mathrm{~b})$ для матрицы $B(t)$, получаем оценку матрицы $B(t)$. Дальнейшее очевидно.

Напомним определение преобразования Фурье-Гаусса (FBI-преобразования по терминологии книги [3]). Это преобразование мы определим формулой

$$
F(\psi \mid \hbar, \sigma, q, p)=\int \exp \left(-\frac{|x-q|^{2} /(2 \sigma)+i(p,(x-q))}{\hbar}\right) \psi(x) d x
$$

Обратное преобразование вычисляется по формуле

$$
\begin{aligned}
\psi(x)=(2 & \pi \hbar)^{-3}(\pi \hbar \sigma)^{-3 / 2} \\
& \times \int \exp \left(-\frac{|x-q|^{2} /(2 \sigma)-i(p,(x-q))}{\hbar}\right) F(\psi \mid \hbar, \sigma, q, p) d q d p .
\end{aligned}
$$


Напомним, что справедливо равенство Парсеваля

$$
\int|\psi(x)|^{2} d x=(2 \pi \hbar)^{-3}(\pi \hbar \sigma)^{-3 / 2} \int|F(\psi \mid \hbar, \sigma, q, p)|^{2} d q d p .
$$

В $L^{2}\left(\mathbb{R}^{3}\right)$ определим оператор $J(k, s, \hbar, \sigma)$ :

$$
\begin{aligned}
J(k, s, \hbar, \sigma) \psi(x)=D_{s}^{3} \int[\exp (i(k, Q)+i s(k, x-Q))] \\
\quad \times W\left(x, t, q_{0}, p_{0}\right) F\left(\psi \mid \hbar, \sigma, q_{0}, p_{0}\right) d q_{0} d p_{0} .
\end{aligned}
$$

Лемма 3. На интервале $0 \leqslant t \leqslant$ аб при $0 \leqslant s \leqslant 1$ в норме пространства $L^{2}\left(\mathbb{R}^{3}\right)$ справедлива оченка

$$
\|J(k, s, \hbar, \sigma)\| \leqslant \text { const } \cdot(1+|k|)^{3}(\hbar \sigma)^{3 / 2} .
$$

ДокАЗАТЕЛьство. Вычисляя преобразование Фурье-Гаусса обеих частей равенства (26), мы получаем

$$
F(J \psi \mid q, p)=\int \omega\left(q, p, q_{0}, p_{0}\right) F\left(\psi \mid q_{0}, p_{0}\right) d q_{0} d p_{0},
$$

где

$$
\begin{aligned}
\omega\left(q, p, q_{0}, p_{0}\right)=(2 \pi \hbar)^{-3}(\operatorname{det}(A(t)))^{-1 / 2} D_{s}^{3}(2 \pi \sigma)^{3 / 2}(\operatorname{det}(E+\sigma C(t)))^{-1 / 2} \\
\times \exp (-\beta(s)) \exp \left(-\left(\frac{\left((q-Q), C(t)(E+\sigma C(t))^{-1}(q-Q)\right)}{2 \hbar}\right.\right. \\
\left.\left.\quad-\frac{i S(t)}{\hbar}+\frac{i(p,(q-Q))}{\hbar}-i(k, Q)\right)\right)
\end{aligned}
$$

и

$$
\beta(s)=\frac{\sigma\left((P-p+s \hbar k),(E+\sigma C(t))^{-1}(P-p+s \hbar k)\right)}{2 \hbar}
$$

Из (30) и (22) следуют оценки

$$
\begin{aligned}
& \left|\beta^{\prime}(s)\right| \leqslant \text { const } \cdot(1+|k|)(|q-Q|+\sigma|P-p+s \hbar k|), \\
& \left|\beta(s)^{\prime \prime}\right| \leqslant \text { const } \cdot(1+|k|)^{2} \hbar \sigma, \\
& \operatorname{Re} \beta(s) \geqslant \frac{\delta \sigma|P-p+s \hbar k|^{2}}{\hbar}, \\
& \operatorname{Re}\left((q-Q), C(t)(E+\sigma C(t))^{-1}(q-Q)\right) \geqslant \frac{\delta|q-Q|^{2}}{\sigma} .
\end{aligned}
$$

Поэтому

$$
\begin{aligned}
\mid \omega(q, & \left.p, q_{0}, p_{0}\right) \mid \leqslant \text { const } \cdot \hbar^{-3}(1+|k|)^{3} \\
& \times\left[\hbar \sigma(|q-Q|+\sigma|P-p+s \hbar k|)+|q-Q|^{3}+\sigma^{3}|P-p+s \hbar k|^{3}\right] \\
& \times \exp \left(-\delta\left(\frac{|q-Q|^{2}}{\hbar \sigma}+\frac{\sigma|P-p+s \hbar k|^{2}}{\hbar}\right)\right) .
\end{aligned}
$$


Из (28), (32) и оценки Карлемана нормы интегрального оператора в $L^{2}\left(\mathbb{R}^{6}\right)$ (с учетом равенства $d q_{0} d p_{0}=d Q d P$ ) следует оценка

$$
\left\|F(J \psi \mid \cdot) \mid L^{2}\left(\mathbb{R}^{6}, d q d p\right)\right\| \leqslant \text { const } \cdot(1+|k|)^{3}(\hbar \sigma)^{3 / 2}\left\|F(\psi \mid \cdot) \mid L^{2}\left(\mathbb{R}^{6}, d q_{0} d p_{0}\right)\right\| .
$$

Из (33) и равенства Парсеваля (25) следует утверждение леммы 3.

В $L^{2}\left(\mathbb{R}^{3}\right)$ определим оператор $R(t)$ :

$$
R(t) \psi(x)=\int\left[V_{2}(Q, x)-V(x)\right] W\left(x, t, q_{0}, p_{0}\right) F\left(\psi \mid \hbar, \sigma, q_{0}, p_{0}\right) d q_{0} d p_{0} .
$$

Лемма 4. На интервале $0 \leqslant t \leqslant$ аб в норме пространства $L^{2}\left(\mathbb{R}^{3}\right)$ справедлива оченка

$$
\|R(t)\| \leqslant \text { const } \cdot(\hbar \sigma)^{3 / 2} .
$$

ДокАЗАТЕЛЬство. Используя известное выражение для остаточного члена формулы Тейлора и (3), мы получаем

$$
\begin{aligned}
V_{2}(Q, x) & -V(x)=-\frac{1}{6} \int_{0}^{1}(1-s)^{3} D_{s}^{3} V(Q+s(x-Q)) \\
=- & \frac{1}{6} \int_{0}^{1}(1-s)^{3}\left(\widehat{v_{\operatorname{ext}}(k)} D_{s}^{3} \exp (i(k, Q+s(x-Q))) d k\right) d s \\
& -\frac{1}{6} \int_{0}^{1}(1-s)^{3}\left(\sum_{j} a(j) D_{s}^{3} \exp (i(b(j), Q+s(x-Q)))\right) d s .
\end{aligned}
$$

Подставив (36) в (34), мы получим

$$
\begin{aligned}
R(t) \psi(x)= & \left.-\frac{1}{6} \int_{0}^{1}(1-s)^{3}\left(\int \widehat{v_{\text {ext }}(k)} J(k, s, \hbar, \sigma) \psi(x)\right] d k\right) d s \\
& -\frac{1}{6} \int_{0}^{1}(1-s)^{3}\left(\sum_{j} a(j) J(b(j), s, \hbar, \sigma) \psi(x)\right) d s .
\end{aligned}
$$

Теперь мы воспользуемся оценкой (27) и условием (4). Получим

$$
\|R(t) \psi\| \leqslant \text { const } \cdot \operatorname{norm}(v)(\hbar \sigma)^{3 / 2}\|\psi\| .
$$

Лемма 4 доказана.

\section{§ 3. Оценка функции Грина}

В $L^{2}\left(\mathbb{R}^{3}\right)$ определим оператор $K(t, \hbar, \sigma)$ :

$$
K(t, \hbar, \sigma) \psi(x)=\int W\left(x, t, q_{0}, p_{0}\right) F\left(\psi \mid \hbar, \sigma, q_{0}, p_{0}\right) d q_{0} d p_{0} .
$$

Положим

$$
U(t, \hbar)=\exp \left(-\frac{i t H}{\hbar}\right)
$$


ТЕОРема 1. Если потенииал v $(x)$ удовлетворяет условиям (3), то для любых $T<\infty, \sigma>0$ существуют такое $N(T)$ и такая константа $C(T)$, что при всех $n>N(T), 0<\hbar<\hbar_{0}, 0<t<T$ в норме пространства $L^{2}\left(\mathbb{R}^{3}\right)$ справедлива оченка

$$
\left\|K\left(\frac{t}{n}, \hbar, \frac{\sigma}{n}\right)^{n}-U(t, \hbar)\right\|<C(T) \hbar^{1 / 2} n^{-3 / 2}
$$

ДОКАЗАТЕЛЬСТво. Положим

$$
\psi(x, t)=K(t, \hbar, \sigma) \psi_{0}(x)
$$

Умножая обе части уравнения (9a) и начальные условия (9b) на $F\left(\psi_{0} \mid \hbar, \sigma, q_{0}, p_{0}\right)$, интегрируя по $d q_{0} d p_{0}$ и учитывая формулу обращения (24), мы получаем, что функция $\psi(x, t)$ есть решение задачи

$$
i \hbar \partial_{t} \psi(x, t)=H \psi(x, t)+R(t) \psi(x, t), \quad \psi(x,+0)=\psi_{0}(x),
$$

откуда следует, что

$$
K(t, \hbar, \sigma)=U(t, \hbar)+\frac{1}{i \hbar} \int_{0}^{t} U(t-\tau, \hbar) R(\tau) d \tau
$$

Из (41) и леммы 4 следует оценка

$$
\|K(t, \hbar, \sigma)-U(t, \hbar)\| \leqslant \mathrm{const} \cdot \hbar^{1 / 2} \sigma^{5 / 2}, \quad 0 \leqslant t \leqslant a \sigma .
$$

Из (42) следует, что существует такое $N(T)<\infty$, что при $n>N(T), 0<t<T$ справедлива оценка

$$
\left\|K\left(\frac{t}{n}, \hbar, \frac{\sigma}{n}\right)-U\left(\frac{t}{n}, \hbar\right)\right\| \leqslant \text { const } \cdot n^{-5 / 2}
$$

Запишем равенство

$$
K\left(\frac{t}{n}, \hbar, \frac{\sigma}{n}\right)^{n}=\left(U\left(\frac{t}{n}, \hbar\right)+\left(K\left(\frac{t}{n}, \hbar, \frac{\sigma}{n}\right)-U\left(\frac{t}{n}, \hbar\right)\right)\right)^{n}=U(t, \hbar)+[\cdots],
$$

где через $[\cdots]$ мы обозначили сумму слагаемых, каждое из которых содержит хотя бы один множитель $(K(t / n, \hbar, \sigma / n)-U(t / n, \hbar))$. В силу унитарности оператора $U(t, \hbar)$ и оценки (43) из (44) следует оценка

$\left\|K\left(\frac{t}{n}, \hbar, \frac{\sigma}{n}\right)^{n}-U(t, \hbar)\right\|=\|[\cdots]\| \leqslant\left(1+\text { const } \cdot \hbar^{1 / 2} n^{-5 / 2}\right)^{n}-1 \leqslant C(T) \hbar^{1 / 2} n^{-3 / 2}$.

Теорема доказана. 
Формула (40) сводит решение задачи (5) к итерации интегрального оператора

$$
\begin{aligned}
& F(\psi \mid \hbar, \sigma q, p) \mapsto F((K \psi) \mid \hbar, \sigma q, p)=2^{-3 / 2}(\pi \hbar)^{-3} \sigma^{3 / 2} \int(\operatorname{det}(A+\sigma B))^{-1 / 2} \\
& \quad \times \exp \left(-\frac{1}{\hbar}\left(\frac{\sigma\left((P-p),(E+\sigma C)^{-1}(P-p)\right)}{2}\right.\right. \\
& \quad+\frac{\left((q-Q), C(E+\sigma C)^{-1}(q-Q)\right)}{2} \\
& \left.\left.\quad-i\left((q-Q),(E+\sigma C)^{-1}(P-p)\right)-i(p,(q-Q))-i S(t)\right)\right) \\
& \quad \times F\left(\psi \mid \hbar, \sigma q_{0}, p_{0}\right) d q_{0} d p_{0} .
\end{aligned}
$$

Ядро интегрального оператора (45) есть сглаженная $\delta$-функция в окрестности классической траектории. Это обстоятельство позволяет компенсировать усложнения, связанные с увеличением размерности фазового при переходе к FBI-преобразованию и на основе формулы (40) построить эффективный алгоритм численного решения задачи (5) (см. [18]-[20]). Заметим, что вычисления по формулам (40) или (45) дают приближение решения сплайном Габора. Это обстоятельство существенно упрощает вычисление матричных элементов и матрицы плотности. Без усложнения рассуждений и выкладок к потенциалу $v(x)$ можно было бы добавить квадратичный по $x$ полином. Аналогичные рассуждения приведены в [21], и мы не будем на этом останавливаться.

\section{Список литературы}

[1] D. Fujiwara, "A construction of the fundamental solution for the Schrödinger equations", J. Anal. Math., 35 (1979), 41-96.

[2] D. Fujiwara, "Remarks on convergence of the Feynman path integrals", Duke Math. J., 47:3 (1980), 559-600.

[3] G. B. Folland, Harmonic analysis in phase space, Princeton Univ. Press, Princeton, NJ, 1989.

[4] S. H. Fricke, A. B. Balantekin, T. Uzer, "Uniform approximation and coherent state path integrals", J. Math. Phys., 32:11 (1991), 3125-3129.

[5] Wei-Min Zhang, Da Hsuan Feng, R. Gilmore, "Coherent states: Theory and some applications", Rev. Modern Phys., 62:4 (1990), 867-927.

[6] M.A. de Gosson, Extended Weyl calculus and application to the phase-space Schrödinger equation, arXiv: math.SG/0503709.

[7] M.A. de Gosson, Symplectically covariant Schrödinger equation in phase space, arXiv: math-ph/0505073.

[8] G. A. Hagedorn, "Semiclassical quantum mechanics: The $\hbar \rightarrow 0$ limit for coherent states", Comm. Math. Phys., 71:1 (1980), 77-93.

[9] G. A. Hagedorn, "Raising and lowering operators for semiclassical wave packets", Ann. Phys., 269:1 (1998), 77-104.

[10] G. A. Hagedorn, A. Joye, "Exponentially accurate semiclassical dynamics: Propagation, localization, Ehrenfest times, scattering, and more general states", Ann. Henri Poincaré, 1:5 (2000), 837-883.

[11] M. F. Herman, "Dynamics by semiclassical methods", Annu. Rev. Phys. Chem., 45 (1994), 83-111. 
[12] S.L. Robinson, "Semiclassical mechanics for time-dependent Wigner functions", J. Math. Phys., 34:6 (1993), 2185-2205.

[13] В.В.Додонов, В.И.Манько, "Инварианты и эволюция нестационарных квантовых систем", Тр. Ин-та физики АН СССР, 183 (1987), 182-286.

[14] J. Bolte, R. Glaser, "A semiclassical Egorov theorem and quantum ergodicity for matrix valued operators", Comm. Math. Phys., 247:2 (2004), 391-419; arXiv: math-ph/ 0204018.

[15] A. Martinez, V. Sordoni, "Microlocal WKB expansions", J. Funct. Anal., 168:2 (1999), 380-402.

[16] D. Chruściński, K. Młodawski, "Wigner function and Schrödinger equation in phasespace representation", Phys. Rev. A, 71 (2005), 052104; arXiv: quant-ph/0501163.

[17] T. Ichinose, S. Takanobu, "Estimate of the difference between the Kac operator and the Schrödinger semigroup", Comm. Math. Phys., 186:1 (1997), 167-197.

[18] Г. Р. Айдагулов, "Применение преобразования Фурье-Гаусса к решению задачи Коши для уравнения Шрёдингера", ЖВМ и МФ, 42:12 (2002), 1810-1815.

[19] Г.Р. Айдагулов, "Применение преобразования Фурье-Гаусса к решению задачи Коши для уравнения Шрёдингера: теоретический анализ численного алгоритма", Вычислит. методы и програм., 4:2 (2003), 16-20; http://num-meth.srcc.msu.su/ zhurnal/tom_2003/v4r119.html.

[20] Г.Р. Айдагулов, "Метод подвижной сетки для решения нестационарного уравнения Шрёдингера", Вычислит. методы и програм., 5 (2004), 18-30; http://num-meth.srcc.msu.su/zhurnal/tom_2004/v5r102.html.

[21] F. Treves, "Parametrices for a class of Schrödinger equations", Comm. Pure Appl. Math., 48:1 (1995), 13-78.

А. А. Арсеньев (А. А. Arsen'ev)

Московский государственный университет

им. М. В. Ломоносова

E-mail: a_arsenev@mail.ru
Поступила в редакцию 26.12 .2005 